Als Einheit der Helligkeit ist jetzt die bei seiner photographischen Wiederauffindung in Heidelberg am 22. März $(\log r=0.7555, \log \Delta=0.6965)$ zugrunde gelegt. Die Größe desselben wurde zu 13.8 und bei einer zweiten Aufnahme am 5. April zu 13.6 angegeben; seine Größe warde sich darnach Anfang August auf 14.8 stellen. Er sollte daher um jene Zeit nicht nur photographisch, sondern mit unseren stärksten Refraktoren auch visuell noch beobachtet werden können.

Eine möglichst lange Verfolgung dieses Kometen wäre aber nicht nur schon an und far sich recht interessant, sondern in einer gewissen Beziehung auch wichtig. Kurz vor der nächsten Opposition, die in der letzten Hälfte des Mai 1908 eintritt, steigt seine (theoretische) Helligkeit wieder auf 0.43 derjenigen an, auf die er am 4 . August 1907 herab-

Wien, I 907 Mai 12. gesunken sein wird, wie folgende rohe Berechnung einer Position um jene Zeit erkennen läßt:

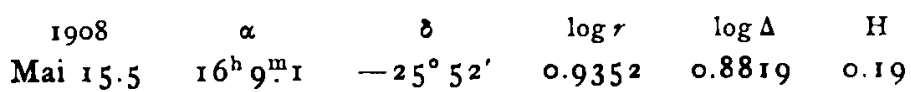
wo, wie oben, die Helligkeit von 1907 März 23 als Einheit zugrunde liegt.

Eine Verfoigung des Kometen bis in den Juli hinein, würde nun einen guten Anhaltspunkt dafur bieten, zu beurteilen, ob es nicht am Ende gar möglich sein könnte, das Gestirn im Jahre 1908 bei seinem Heraustreten aus den Sonnenstrahlen nochmals wiederzufinden. Ich ersuche Idaher die Herren, denen die entsprechenden Instrumente zur Disposition stehen, freundlichst um möglichst langes Verfolgen dieses Kometen.

E. Weiß.

\title{
Ein zweiter kleiner Planet der Jupitergruppe 1907 XM.
}

Auf Wunsch von Herrn Prof. Kreutz habe ich es unternommen, die Bahn des von Kopff in Heidelberg am ro. Febr. dieses Jahres entdeckten Planeten 1907 XM zu berechnen. Schon eine erste vorlaufige Bahnberechnung aus den Beobachtungen Febr. 10, März 3 und März 2 I zeigte, daß die mittlere Bewegung von 1907 XM von der mittl. Bewegung des Planeten Jupiter nicht beträchtlich abweichen konnte. Nachher habe ich nach der Methode der Variation der Distanzen, unter exakter Darstellung der beiden äußersten Örter, aus den Beobachtungen Königstuhl Febr. 10, Wien März 11,21 und April 1 2, 16 eine neue Bahn gerechnet. Die Elemente lauten:

Epoche 1907 Febr. 10.0 M. Z. B.

$$
\begin{aligned}
& M=335^{\circ} 47^{\circ} 2^{\prime \prime} \cdot 3 \\
& \omega=183 \text { 5 I } 5 \text { I.9 } \\
& \delta=3415^{8} \quad 24.9 \text { I907.0 } \\
& i=187 \times 6.9 \\
& \begin{aligned}
\varphi & =2^{\circ} 8^{\prime} 23^{\prime \prime} 6 \\
\mu & =292^{\prime \prime} 5^{84} \\
\log a & =0.722504
\end{aligned}
\end{aligned}
$$

und der Vergleich dieser Elemente mit sämtlichen Beobachtungen gibt in $\lambda$ und $\beta$ die Abweichungen $(B-R)$ :

$$
\begin{aligned}
& \text { r } 907 \text { Febr. 10*) Königstuhl - I."o +2."2 } \\
& \text { März } 3 \text { - } 2.3+0.1 \\
& 7 \text { Wien }-1.6+1.3 \\
& \text { II } \quad-0.2+0.8 \\
& 2 \mathrm{I} \quad+0.2 \quad-0.8 \\
& \text { April } 12 \text { +2.1 }+0.9 \\
& \text { I } 6 \text { * }-0.1 \quad 0.0 \\
& 19 \text {, }+2.8+8.3
\end{aligned}
$$

Wir haben also hiermit einen zweiten Jupiter-nahen kleinen Planeten erhalten. Ob die Bewegung dieses Körpers irgend einen Zusammenhang hat mit den von mir bei der ersten Nachricht über die eventuelle Identität der mittleren Bewegung Hyperions und der des zehnten Saturnsatelliten (A.N. 4015) und von Prof. Charlier in bezug auf den Planeten (588) [r $906 \mathrm{TG}$ (A. N. 4094) erwähnten Lagrangeschen Sätzen, ist schwierig zu sagen. In diesem Falle ist die Elongation rom Dreieckspunkt beträchtlich $\left(19^{\circ}\right)$, und außerdem ist die Bahnneigung ziemlich groß.

Kiel, Bureau der Astr. Nachr., 1907 Mai 7 .
Die bis jetzt erhaltenen theoretischen Resultate uber die Bewegung in der Nähe von den Librationspunkten $L_{4}$ und $L_{3}$ (den Dreieckspunkten) geben ja nur eine erste Näherung, die im Falle beträchtlicher Abweichung von diesen Librationspunkten nicht einmal eine Näherung darstellen. Insbesondere darf in bezug auf die Stabilität hierher gehörender Bewegungsformen nicht außer acht gelassen werden, $\mathrm{da} ß$, auch wenn man durch strenge Methoden die Existenz rein periodischer Bewegungen ableiten kann, die ganzen Resultate dadurch illusorisch werden, $\mathrm{da} B$ die Störungen seitens der anderen Planeten unseres Sonnensystems den strengen Charakter einer solchen Bewegung zerstören werden; hierin liegt überhaupt der schwache Punkt der Anwendung der Theorie der periodischen Lösungen für konkrete astronomische Probleme.

So viel durfte jedenfalls sicher sein, daß ein kleiner Planet mit derselben oder sehr nahe derselben mittleren Bewegung wie Jupiter sich lange Zeiten hindurch in der Nähe von einem der Dreieckspunkte erhalten kann, da die Störun. gen seitens Jupiter hier außerordentlich klein sind und wegen der großen Entfernungen - auch die anderen Körper des Sonnensystems sehr lange Zeiten brauchen, um einen beträchtlichen Einfluß auszuluben. Ich hoffé Gelegenheit zu haben auf diesen Punkt zuruckzukommen.

Die Entdeckung der zwei interessanten neuen Planeten, r 906 TG und 1907 XM, führt auch die Gedanken auf eine andere Frage, die ein großes Interesse beanspruchen darf. Haben wir hier nur mit einer abgeschlossenen Gruppe von Himmelskörpern zu tun, die durch Jupiter far lange Zeiten in der Nähe der Jupiterbahn festgehalten werden, oder sind diese Neuentdeckungen als Vorboten zu einer Erweiterung des Systems der kleinen Planeten uber die Jupiterregion hinaus aufzufassen? A priori ist ja diese letztere Möglichkeit nicht von der Hand zu weisen; das Suchen mit den zur Verfutgung stehenden großen Fernrohren wird wohl in nicht allzu ferner Zukunft die Antwort geben.

*) Daß diese Beobachtung nicht strenge dargestellt wird, ribbrt daher, da $B$ ich bei der Ableitung der Elemente eine ältere, etwas andere Werte gebende Ausmessung der Platte benutzt hatte. 